\title{
Aripiprazole for sexual disinhibition in frontotemporal dementia
}

\author{
A. Jorge ${ }^{1}$, J. Carvalheiro', L. Paiva Delgado ${ }^{1}$ \\ ${ }^{1}$ Centro Hospitalar do Médio Tejo, Psychiatry, Tomar, Portugal.
}

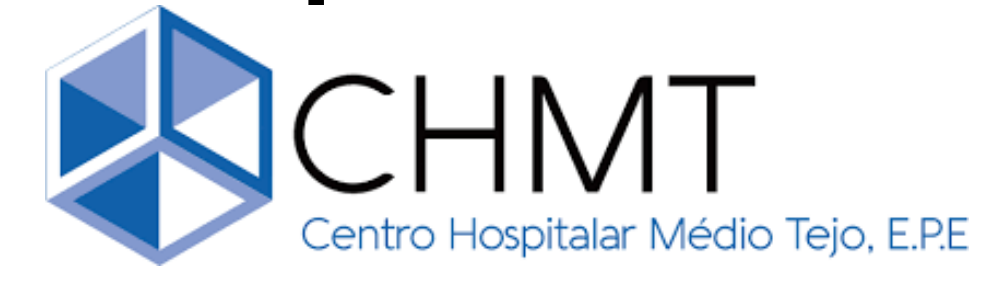

\section{Background and Aims:}

Frontotemporal dementia (FTD) is the second most common cause of young-onset dementia and is associated with pervasive changes in behavior and personality.

Characteristic changes in FTD include alterations in eating, emotion processing, and sleeping.

Sexual disinhibition is commonly reported in the behavioral variant of FTD (bvFTD) and has led to the assumption that bvFTD patients exhibit hypersexual behavior. The management of this behavior can be difficult.

\section{Objectives:}

To do a non systematic review about this issue.

\section{Materials and Methods:}

Pubmed and medscape was used as bibliographical sources.

\section{Results:}

Sexual disinhibition may be regarded as sexually inappropriate or "acting out" behavior.

Impulsive behavior and poor judgment often occur in dementia, and inappropriate sexual impulses that were once controlled by intact judgment or moral values may emerge. Such behaviors have been reported more commonly in men. Some examples include genital exposure, public masturbation, propositions to others for sexual intercourse, and attempts at coitus or fondling another person's genitals or breasts.

The evidence to date has led to the conclusion that sexual disinhibition and aberrant behavior is a common behavioral manifestation of bvFTD. There are reports of sexual disinhibition behavior among 8-18\%\% of bvFTD patients.

The sexual symptoms result in significant personal distress or impairment in social, occupational or other important areas of functioning.

Dementia patients with frontal lobe dysfunction often react impulsively to tempting environmental situations involving sexual or other objects of interest without concern for the consequences.

Sexual disinhibition as part of a frontally-mediated general disinhibition, however, does not explain hypersexuality due to an increased sexual drive.

\section{Management}

There exist both nonpharmacological and pharmacological approaches for the treatment sexual disinhibition.

It is usually accepted as appropriate to begin with nonpharmacological approaches in order to avoid unnecessary adverse effects.
Nonpharmacological treatment can be divided into environmental, behavioral and educational. These interventions should involve not only the patients but also families and the nursing staff for institutionalized subjects, bearing in mind that the aim is to try and promote an appropriate manifestation of sexual behavior.

If the nonpharmacological approach is unsuccessful, pharmacological intervention may then be necessary.

Case reports have described the use of a wide variety of medications in the male population, including antidepressants, gonadotropin releasing hormone analogues, antiandrogens, estrogens, anticonvulsants, antipsychotics, and antihistamines.

Atypical antipsychotics are frequently used for the treatment of dementia related psychosis. Sexual disinhibition is one of the most difficult group of symptoms to manage.

In the literature, there are some frontotemporal dementia cases with sexual disinhibition, treated successfully with aripiprazole.

In one of the cases, sertraline and paroxetine were reported to be ineffective, but administering aripiprazole at $30 \mathrm{mg} /$ day for 2 weeks markedly improved the patient's condition.

In another FTD patient, aripiprazole was initiated at 12 $\mathrm{mg} / \mathrm{day}$, and the dose was gradually increased to $18 \mathrm{mg} / \mathrm{day}$ in a week. Two weeks after the start of the medication, all of the sexual symptoms subsided.

\section{Conclusions:}

Hypersexual behaviors in a patient can interfere with the therapeutic alliance and create a burden for staff, other residents, and family members.

Although treatment of sexual disinhibition in FTD with aripiprazole is currently an off-label use, some cases showed a benefic effect of this molecule.

Further reports are expected in the future regarding the optimum dose and mechanism of action in patients with this type of dementia.

Sources: 1. Sarikaya, S., \& Sarikaya, B. (2018). Aripiprazole for the Treatment of Inappropriate Sexual Behavior: Case Report of an Alzheimer's Disease Patient Known as Heterosexual with Recently Shifted Sexual Orientation to Same Gender. Journal of Alzheimer's Disease Reports, 2(1), 117-121. 2. Nomoto, H., Matsubara, Y., Ichimiya, Y., \& Arai, H. (2017). A case of frontotemporal dementia with sexual disinhibition controlled by aripiprazole. Psychogeriatrics, 17(6), 509-510. 3. Mendez, M. F., \& Shapira, J. S. (2013). Hypersexual Behavior in Frontotemporal Dementia: A Comparison with EarlyOnset Alzheimer's Disease. Archives of Sexual Behavior, 42(3), 501-509. 4. Reeves, R. R., \& Perry, C. L. (2013). Aripiprazole for Sexually Inappropriate Vocalizations in Frontotemporal Dementia. Journal of Clinical Psychopharmacology, 33(1), 145-146 